\title{
COMPOSIÇÃO QUÍMICA DE UM RESÍDUO ALCALINO DA INDÚSTRIA DE PAPEL E CELULOSE (DREGS)
}

Henrique Cesar Almeida*, Cristian Berto da Silveira, Paulo Roberto Ernani, Mari Lucia Campos e Denice Almeida Departamento de Solos, Centro de Ciências Agroveterinárias, Universidade do Estado de Santa Catarina, Av. Luiz de Camões, 2090, 88520-000 Lages - SC, Brasil

Recebido em 17/10/06; aceito em 2/3/07; publicado na web em 30/7/07

\begin{abstract}
CHEMICAL COMPOSITION OF DREGS. Dregs is an alkaline solid by-product generated in the cellulose manufacturing industry that could be used to correct soil acidity. The present study aimed to evaluate the chemical composition of this product and some of its properties. The dregs presented $354 \mathrm{~g} \mathrm{~kg}^{-1}$ of calcium, neutralization capacity of $80.3 \%$, and $\mathrm{pH} 10.7$, besides low concentration of sodium $\left(10.2 \mathrm{~g} \mathrm{~kg}^{-1}\right)$, lead $\left(62.9 \mathrm{mg} \mathrm{kg}^{-1}\right)$ and cadmium $\left(5.6 \mathrm{mg} \mathrm{kg}^{-1}\right)$. Thus, it is a product that can safely be used to increase the soil $\mathrm{pH}$.
\end{abstract}

Keywords: industrial residue; soil acidity amendment; heavy metals.

\section{INTRODUÇÃO}

Durante os processos industriais são gerados muitos resíduos, podendo ser de origem sólida, líquida ou gasosa. Todos são de grande importância ambiental, porém os de origem sólida, que apresentam reatividade e ocupam grande espaço físico, devem receber um cuidado especial. Existem três técnicas principais para tratamento dos resíduos sólidos: incineração, redução da toxicidade, e disposição no solo. Esta última depende de fatores geográficos, geológicos e climáticos, além da potencialidade de reaproveitamento ${ }^{1}$. Neste sentido, o uso desses resíduos na agricultura tornou-se bastante comum devido à capacidade do solo de inativar quimicamente diversos compostos, principalmente os metais, que são retirados da solução do solo e retidos pela fase sólida por mecanismos de sorção ${ }^{2}$. Com o surgimento de novos sistemas de tratamentos, que reduzem sensivelmente o potencial poluidor dos resíduos, o uso agrícola, seja como fonte de nutrientes para as plantas ou como corretivo da acidez do solo, dependendo da composição química dos mesmos, tem-se tornado uma alternativa barata e interessante na preservação da qualidade ambiental ${ }^{3,4}$. No entanto, ao se recomendar tal utilização, é necessário estudar possíveis alterações que possam ocorrer nas propriedades físico-químicas do solo, bem como a resposta das plantas a tais produtos 5 .

A lama de cal e o dregs são dois resíduos utilizados como corretivos da acidez do solo. Ambos são derivados do processo de separação da celulose, que é extraída da madeira por meio de ataque alcalino ${ }^{6}$. Neste processo, são utilizados reagentes oxidantes e alcalinos que compõem o chamado licor branco $\left(\mathrm{NaOH}+\mathrm{Na}_{2} \mathrm{~S}-\right)^{7}$. Da reação do licor branco com a madeira originam-se dois subprodutos: a polpa celulósica e o licor negro. Este último, após ser evaporado a teor de sólidos, é queimado na caldeira de recuperação, resultando em um fundido, que logo após a dissolução com o licor branco fraco proveniente da lavagem da lama de cal, forma o chamado licor verde $\left(\mathrm{Na}_{2} \mathrm{CO}_{3}+\mathrm{Na}_{2} \mathrm{SO}_{4}+\mathrm{Na}_{2} \mathrm{~S}+\mathrm{Fe}(\mathrm{OH})_{2}\right)^{7}$. Da caustificação do licor verde, ou seja, após a adição de óxido de cálcio $(\mathrm{CaO})$, é extraída a lama de cal, um resíduo de coloração branca formado predominantemente por carbonato de cálcio $\left(\mathrm{CaCO}_{3}\right)$. O dregs, por sua vez, possui cor acinzentada e é removi-

*e-mail: henriquecav@gmail.com do durante a clarificação do licor verde, ou seja, por ocasião da remoção de impurezas (carbono, partículas de lama, hidróxidos e sulfetos de metais, além de outros elementos).

Os valores de neutralização (VN) destes resíduos são proporcionais aos teores de óxidos, hidróxidos e carbonatos associados quimicamente a elementos como o $\mathrm{Ca}$ e o $\mathrm{Na}$. Estes valores podem variar de 40 a $70 \%$ para a lama de cal e é de aproximadamente $40 \%$ para o licor verde ${ }^{8,9}$. O VN é a capacidade neutralizante do corretivo de acidez do solo, índice que faz parte do Poder Relativo de Neutralização Total (PRNT) ${ }^{10}$. O PRNT também considera o grau de moagem do corretivo (ER), sendo ambos expressos em percentagem de equivalência ao carbonato de cálcio $\left(\mathrm{CaCO}_{3}\right)$ puro, que é de $100 \%{ }^{11}$. Sua fórmula é descrita da seguinte maneira:

\section{$\operatorname{PRNT}(\%)=(\mathrm{VN} \times \mathrm{ER}) / 100$}

Em estudos realizados com o licor verde gerado em outros países, verifica-se a existência de alguns elementos essências às plantas, como cálcio, magnésio, potássio, cobre e zinco, além de atributos importantes, como o valor de neutralização9. No entanto, as informações existentes sobre a composição química do dregs produzido nas indústrias brasileiras se referem a trabalhos realizados na década de $80^{12}$.

O dregs é um resíduo de origem industrial e, como tal, pode ser uma fonte de metais pesados, trazendo grande preocupação quanto a sua utilização na adubação de plantas empregadas diretamente na alimentação humana ${ }^{13}$, assim como na contaminação dos lençóis freáticos ${ }^{14}$. A maior ou menor mobilidade destes metais no solo vai depender de várias características intrínsecas do solo, principalmente o $\mathrm{pH}^{15}$ e o número de sítios de adsorção ${ }^{2}$. Além disso, mesmo em quantidades-traço, alguns destes elementos são muito tóxicos, podendo aumentar este efeito na medida em que vão sendo incorporados ao solo ${ }^{16}$. Neste sentido, o presente trabalho teve por objetivo caracterizar quimicamente o dregs quanto aos teores totais de $\mathrm{Ca}, \mathrm{Mg}, \mathrm{Na}, \mathrm{K}, \mathrm{Fe}, \mathrm{Cu}, \mathrm{Mn}, \mathrm{Zn}, \mathrm{Cd}$, $\mathrm{Pb}, \mathrm{pH}$ e valor de neutralização, verificando assim o potencial de uso do mesmo como corretivo da acidez do solo e como fonte de nutrientes às plantas, além do risco de contaminação por elementos-traço. A análise térmica diferencial e a técnica de espectroscopia no infravermelho foram utilizadas para auxiliar o processo de caracterização química. 


\section{PARTE EXPERIMENTAL}

\section{Local e amostragem}

O trabalho foi conduzido em laboratório, no Centro de Ciências Agroveterinárias da Universidade do Estado de Santa Catarina, durante o primeiro semestre de 2006. O dregs foi coletado no depósito de uma empresa de papel e celulose por meio de trator acoplado com pá carregadeira, até completar o volume de $5 \mathrm{~m}^{3}$. Destes, foram retiradas 5 sub-amostras em 5 pontos do lote, formando uma amostra composta de $200 \mathrm{~g}$ para as análises químicas.

\section{Atributos químicos do resíduo}

Os atributos químicos analisados foram $\mathrm{pH}$ e valor de neutralização (VN). Para a determinação do VN, utilizou-se $1,00 \mathrm{~g}$ do produto, ao qual foram adicionados $25 \mathrm{~mL}$ de $\mathrm{HCl} 1,0 \mathrm{~mol} \mathrm{~L}^{-1}$. A mistura foi aquecida em banho-maria a $90 \pm 5{ }^{\circ} \mathrm{C}$ por $30 \mathrm{~min}$. Após isso, adicionaram-se mais $30 \mathrm{~mL}$ de água destilada deixando a mistura ferver em placa de aquecimento por $5 \mathrm{~min}$, seguido de resfriamento por $1 \mathrm{~h}$. A seguir, a mistura foi homogeneizada em um balão volumétrico de $100 \mathrm{~mL}$, completou-se o volume e deixou-se decantar durante 2 a $3 \mathrm{~h}$. Por último, $50 \mathrm{~mL}$ do sobrenadante foram transferidos para erlenmeyer de $125 \mathrm{~mL}$ e a seguir titulados com $\mathrm{NaOH}$ 0,5 mol L-1, usando-se fenolftaleina $1,0 \%$ como indicador. $\mathrm{O}$ pH foi determinado por meio de potenciometria, na relação resíduo:água destilada de 1:1, após agitação seguida de $30 \mathrm{~min}$ de repouso e agitação novamente por ocasião da leitura. As duas metodologias estão descritas em Tedesco et al. ${ }^{10}$.

\section{Análise química dos elementos}

Foram feitas determinações de $\mathrm{Ca}, \mathrm{Mg}, \mathrm{K}, \mathrm{Na}, \mathrm{Fe}, \mathrm{Cu}, \mathrm{Zn}, \mathrm{Mn}$, $\mathrm{Cd}$ e $\mathrm{Pb}$. Para as análises dos teores totais desses elementos, foi utilizado o método USEPA 3050B, da Agência de Proteção Ambiental dos $\mathrm{EUA}^{17}$. Essa metodologia consiste em pesar, dentro de tubos de digestão, $0,50 \mathrm{~g}$ do produto passado previamente em peneira com malha de $0,15 \mathrm{~mm}$. A seguir, são adicionados $10 \mathrm{~mL}$ de $\mathrm{HNO}_{3}$ concentrado e os tubos são transferidos para bloco digestor para serem aquecidos durante $10 \mathrm{~min}$ a $95 \pm 5^{\circ} \mathrm{C}$. Após isso, as amostras são resfriadas para receberem a adição de mais $5,0 \mathrm{~mL}$ de $\mathrm{HNO}_{3}$ concentrado e são novamente levadas ao bloco digestor para outro aquecimento por $2 \mathrm{~h}$ na temperatura de $95 \pm 5{ }^{\circ} \mathrm{C}$. Em seguida, são resfriadas, e a elas são adicionados $2 \mathrm{~mL}$ de água destilada e $3 \mathrm{~mL}$ de $\mathrm{H}_{2} \mathrm{O}_{2} 30 \%$, sendo novamente conduzidas ao bloco digestor por mais $2 \mathrm{~h}$ na mesma temperatura $\left(95 \pm 5^{\circ} \mathrm{C}\right)$. A seguir, são resfriadas mais uma vez e são adicionados $5 \mathrm{~mL}$ de $\mathrm{HCl}$ concentrado e 10 $\mathrm{mL}$ de água destilada, quando são aquecidas pela última vez por 5 min na mesma temperatura $\left(95 \pm 5^{\circ} \mathrm{C}\right)$. Finalmente, são resfriadas e armazenadas em recipientes plásticos ${ }^{18}$. As concentrações totais de $\mathrm{Ca}, \mathrm{Mg}, \mathrm{Fe}, \mathrm{Cu}, \mathrm{Zn}, \mathrm{Mn}, \mathrm{Pd}$ e $\mathrm{Cd}$ foram determinadas em um espectrofotômetro de absorção atômica (AA) Perkin - Elmer Analyst 100 , enquanto que os valores de $\mathrm{Na}$ e de $\mathrm{K}$ foram determinados em um fotômetro de emissão Digimed DM - 61.

\section{Espectroscopia no infravermelho}

O espectro de infravermelho foi obtido em espectrofotômetro Perkin - Elmer FT-IR 1600, com sistema de registro computadorizado. A amostra foi dispersa em $\mathrm{KBr}$ e o espectro obtido na região de 4000 a $400 \mathrm{~cm}^{-1}$. A amostra de dregs foi previamente submetida a um tratamento térmico por $4 \mathrm{~h}$ na temperatura de $130{ }^{\circ} \mathrm{C}$ para eliminar o excesso de umidade.

\section{Análise térmica}

O termograma da amostra de dregs foi obtido em analisador térmico Schimadzu, TGA-50, com taxa de aquecimento de $10{ }^{\circ} \mathrm{C}$ por min entre 20 e $900{ }^{\circ} \mathrm{C}$, em atmosfera de nitrogênio.

\section{RESULTADOS E DISCUSSÃO}

\section{Atributos químicos do resíduo}

O dregs apresentou pH de 10,70 \pm 0,02 e valor de neutralização (VN) de 80,3 $\pm 2,3(\%)$. O VN é muito semelhante ao dos calcários de boa qualidade utilizados na agricultura ${ }^{10,19}$ e superior ao da lama de cal, que normalmente varia de 40 a $70 \%{ }^{8}$. Sob esse aspecto, o dregs é um produto que pode ser utilizado para elevar o $\mathrm{pH}$ dos solos agrícolas.

\section{Teores totais dos elementos}

O dregs apresentou uma alta concentração de Ca. O teor deste elemento no produto $(35,4 \%)$ foi aproximadamente 35 vezes maior que o de $\mathrm{Mg}(0,92 \%)$ (Tabela 1$)$. Essa relação é elevada quando comparada à existente nos calcários dolomíticos, que possuem uma média de $28 \%$ de $\mathrm{Ca}$ e de $12 \%$ de $\mathrm{Mg}^{11}$. Uma alta relação $\mathrm{Ca}: \mathrm{Mg}$ pode induzir à falta de $\mathrm{Mg}$ às plantas se o produto for adicionado em grandes quantidades em solos com baixa disponibilidade deste elemento $^{20}$. Isto ocorre devido à competição existente entre $\mathrm{Ca} \mathrm{e}$ Mg pelos sítios de absorção presentes nas membranas plasmáticas das raízes das plantas. $\mathrm{O}$ alto teor de $\mathrm{Ca}$ no dregs deve-se à adição deste elemento na forma de óxido de cálcio $(\mathrm{CaO})$ no processo de caustificação, que ocorre durante a recuperação de produtos químicos utilizados no cozimento da madeira ${ }^{6}$.

As concentrações de $\mathrm{Na}$ e $\mathrm{K}$ no dregs foram baixas. $\mathrm{O}$ teor de $\mathrm{Na}$ foi de aproximadamente $1,0 \%$ (Tabela 1), representando menos da metade dos valores encontrados em outros trabalhos realizados com este resíduo, que variaram de 2 a 4\% $\%^{9,12,21}$. A adição de quantidades moderadas a altas de $\mathrm{Na}$ ao solo não é recomendada, pois o excesso desse elemento pode prejudicar algumas propriedades importantes do mesmo sob o ponto de vista agronômico, incluindo aumentos na dispersão de argila e de substâncias orgânicas ${ }^{22-24}$. A dispersão desses colóides normalmente prejudica a parte física do solo, com efeitos negativos na disponibilidade e no fluxo de água e de ar. A dispersão dos colóides acontece porque o $\mathrm{Na}$, por ter um grande raio hidratado, tem dificuldade de se aproximar das superfícies sólidas do solo carregadas negativamente. Com isso, há um aumento da espessura da dupla camada elétrica, favorecendo a dispersão dos colóides ${ }^{25}$. A concentração de $\mathrm{K}$ foi de aproximadamente $0,1 \%$ (Tabela 1 ), valor similar ao encontrado no lodo de esgoto, um resíduo utilizado como fertilizante do solo ${ }^{26}$.

A concentração de micronutrientes no dregs não foi alta (Tabela 1). Os teores de $\mathrm{Cu}\left(54 \mathrm{mg} \mathrm{kg}^{-1}\right)$ e de $\mathrm{Zn}\left(235 \mathrm{mg} \mathrm{kg}^{-1}\right)$ foram semelhantes aos existentes em outros produtos utilizados como fertilizantes do solo, a exemplo dos adubos fosfatados naturais, cuja concentração aproximada varia de 20 a $50 \mathrm{mg} \mathrm{kg}^{-1}$ para $\mathrm{Cu}$ e de 50 a $600 \mathrm{mg} \mathrm{kg}^{-1}$ para $\mathrm{Zn}^{18}$, porém foram inferiores aos existentes no esterco de suínos, o qual apresentou $1,1 \mathrm{~g} \mathrm{~kg}^{-1}$ de $\mathrm{Cu}$ e $18 \mathrm{~g}$ $\mathrm{kg}^{-1}$ de $\mathrm{Zn}^{27}$. Apesar do dregs não ter apresentado um alto teor de Mn (4,8 $\left.\mathrm{g} \mathrm{kg}^{-1}\right)$, a concentração foi superior à existente no lodo de esgoto de cidades como Jundiaí, Barueri e Franca no estado de São Paulo, que variaram de 230 a $690 \mathrm{mg} \mathrm{kg}^{-1}{ }^{28,29}$. O teor de Fe $(3,0 \mathrm{~g}$ $\mathrm{kg}^{-1}$ ) também foi baixo. A concentração desses quatro elementos, apesar de pequena, confere ao produto um caráter fertilizante.

As concentrações de elementos-traço encontradas no dregs tam- 
bém foram baixas (Tabela 1). Os valores totais de $\mathrm{Cd}\left(5,6 \mathrm{mg} \mathrm{kg}^{-1}\right)$ e de $\mathrm{Pb}\left(63 \mathrm{mg} \mathrm{kg}^{-1}\right)$ ficaram abaixo dos limites de intervenção de solos agrícolas ${ }^{30}$ e da concentração máxima permitida ${ }^{31}$, que são de 10 e $840 \mathrm{mg} \mathrm{kg}^{-1}$, respectivamente. A borra de fosfato ${ }^{3}$, um resíduo industrial de siderurgia também utilizado como fertilizante do solo, e os calcários agrícolas ${ }^{15}$, utilizados para elevar o $\mathrm{pH}$ dos solos, mostraram concentrações de Cd similar à do dregs $(4,0$ e 3,0 mg $\mathrm{kg}^{-1}$, respectivamente). A aplicação de sais de $\mathrm{Cd}$ em solos ácidos do Rio Grande do Sul promoveu o declínio na produção de matéria seca das plantas somente a partir da adição de $100 \mathrm{mg} \mathrm{kg}^{-1} \mathrm{de} \mathrm{Cd}^{32}$.

Tabela 1. Concentrações médias e desvio padrão dos elementos químicos presentes no dregs, e limite de detecção dos equipamentos utilizados (LDE). Médias de 5 repetições

\begin{tabular}{lcc}
\hline Elementos & $\begin{array}{c}\text { Médias com Desvio Padrão } \\
\mathrm{g} \mathrm{kg}^{-1}\end{array}$ & $\begin{array}{c}\mathrm{LDE}^{*} \\
\mathrm{mg} \mathrm{kg}^{-1}\end{array}$ \\
\hline $\mathrm{Ca}$ & $354,1 \pm 25,1$ & 0,4 \\
$\mathrm{Mg}$ & $9,2 \pm 0,7$ & 0,1 \\
$\mathrm{Na}$ & $10,2 \pm 1,6$ & 0,1 \\
$\mathrm{~K}$ & $1,2 \pm 0,2$ & 6,2 \\
$\mathrm{Fe}$ & $3,0 \pm 0,5$ & 0,5 \\
$\mathrm{Mn}$ & $4,8 \pm 0,4$ & 0,07 \\
\hline & $\mathrm{mg} \mathrm{kg}-1$ & \\
\hline $\mathrm{Zn}$ & $235,5 \pm 10,9$ & 0,8 \\
$\mathrm{Cu}$ & $54,3 \pm 2,3$ & 0,05 \\
$\mathrm{~Pb}$ & $62,9 \pm 8,7$ & 0,2 \\
$\mathrm{Cd}$ & $5,6 \pm 0,2$ & 0,5 \\
\hline
\end{tabular}

*LDE $=(\mathrm{SM}-\mathrm{Mbr}) / \mathrm{m}$, onde LDE é o limite de detecção do equipamento, $\mathrm{Mbr}=$ média das provas em branco, $\mathrm{m}=$ inclinação da curva de calibração e $\mathrm{SM}=$ sinal analítico mínimo distinguível ${ }^{36}$.

\section{Espectroscopia no infravermelho}

Analisando o espectro de infravermelho da amostra de dregs (Figura 1) nota-se uma banda de forte intensidade na região de $1428 \mathrm{~cm}^{-1}$ e uma banda de média intensidade em $874 \mathrm{~cm}^{-1}$, que são características do carbonato de cálcio $\left(\mathrm{CaCO}_{3}\right)^{33,34}$, indicando sua presença no produto. A existência de grupamentos sulfatos $\left(\mathrm{SO}_{4}^{2-}\right)$ pode ser observada por uma banda de baixa intensidade na região de $1108 \mathrm{~cm}^{-1}$ e se confirma pela presença de outra banda de baixa intensidade em $618 \mathrm{~cm}^{-1} 34$ (Figura 1). A presença do ânion sulfato favorece a mobilidade vertical do cátion sódio no perfil do solo, uma vez que ele tem uma forte tendência de formar pares ionicamente neutros, aumentando a perda de cátions por lixiviação ${ }^{35}$. Em $3454 \mathrm{~cm}^{-1}$ observa-se uma banda de média intensidade que pode ser atribuída ao estiramento dos grupos hidroxílicos $\left(\mathrm{OH}^{-}\right)^{34}$. A presença de carbonatos e de hidroxilas é responsável pelo alto valor de neutralização do produto.

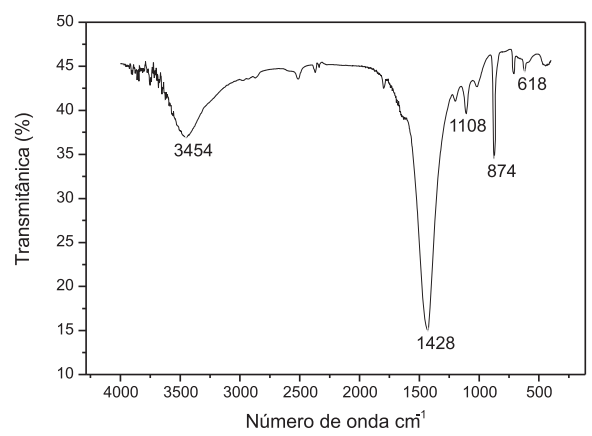

Figura 1. Espectro de infravermelho da amostra de dregs

\section{Análise térmica}

A análise termogravimétrica mostra apenas dois estágios de decomposição térmica (Figura 2). O primeiro, com temperatura inicial em $20,74{ }^{\circ} \mathrm{C}$ e temperatura final em $149^{\circ} \mathrm{C}$, corresponde à perda de água adsorvida pela amostra e a compostos orgânicos presentes no dregs, onde a perda de massa foi de $2,6 \%$. No segundo estágio, verifica-se uma temperatura de decomposição em $753,14{ }^{\circ} \mathrm{C}$, como pode ser observado pela primeira derivada da curva de análise termogravimétrica (DrTGA) (Figura 2). Neste estágio de decomposição térmica, a perda de massa foi de $38,7 \%$, região de decomposição característica dos carbonatos (Equação 1) ${ }^{33}$, o que comprova as análises anteriores. A massa restante da amostra, 58,68\%, apresentou temperatura de decomposição superior a $800{ }^{\circ} \mathrm{C}$, provavelmente devido à presença de outros materiais inorgânicos que são termicamente estáveis desde a temperatura ambiente até $1100{ }^{\circ} \mathrm{C}{ }^{33}$.

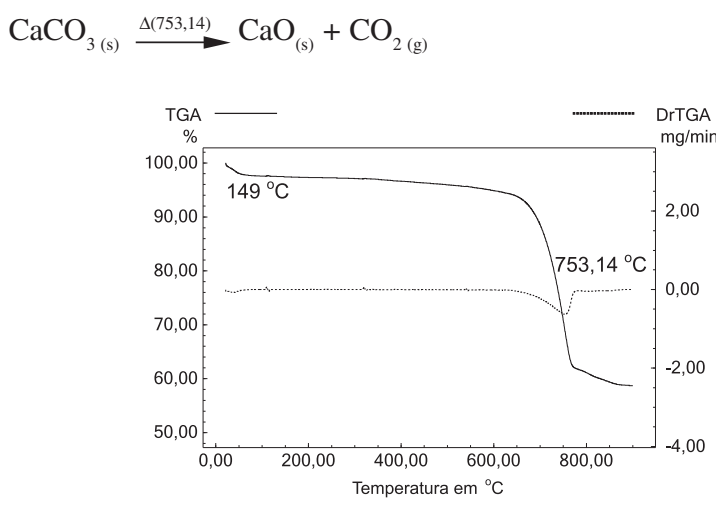

Figura 2. Análise termogravimétrica da amostra de dregs

\section{CONCLUSÕES}

A composição química inorgânica do lote de dregs analisado indica que este produto pode ser utilizado como corretivo da acidez dos solos agrícolas e também como fonte de cálcio e de alguns micronutrientes às plantas, sem riscos de contaminação a curto $\mathrm{e}$ médio prazo.

\section{REFERÊNCIAS}

1. Lora, E. E. S.; Prevenção e controle da poluição dos setores energético, industrial e de transporte, ANEEL: Brasília, 2000.

2. Sparks, D. L.; Environmental soil chemistry, Academic Press: San Diego, 1995.

3. Defelipo, B. V.; Nogueira, A. V.; Loures, E. G.; Alvarez, V. H.; R. bras. Ci. Solo 1992, 16, 127.

4. Mello, S. C.; Vitti, G. C.; Hort. bras. 2002, 20, 452.

5. Ferreira, A. S.; Camargo, F. A. O.; Tedesco, M. J.; Biassini, C. A.; R. bras. Ci. Solo 2003, 27, 755 .

6. Cprh;. Roteiro complementar de licenciamento e fiscalização: indústria de papel e celulose, Companhia Pernambucana do Meio Ambiente: Recife, 1998.

7. Cohn, P. E.; Ribeiro, R. N.; Resumos do $35^{\circ}$ Congresso e Exposição Anual da Celulose e Papel, São Paulo, Brasil, 2002.

8. Suzuki, A.; Basso, C.; Kitazawa, I. H.; Agrop. catarinense 1991, 4, 9.

9. Nurmesniemi, H.; Pöykiö, R.; Perämäki, P.; Kuokkanen, T.; Chemosphere 2005, 61, 883.

10. Tedesco, M. J.; Gianelo, C.; Bissani, C. A.; Bohnen, H.; Wolkweiss, S. J.; Análises de solos, plantas e outros materiais, $2^{\mathrm{a}}$ ed., UFRGS: Porto Alegre, 1995.

11. Quaggio, J. A.; Acidez e calagem em solos tropicais, Instituto Agronômico: Campinas, 2000.

12. Waldemar, C. C.; Herrera, J.; Resumos do $19^{\circ}$ Congresso Anual da ABCP, São Paulo, Brasil, 1986.

13. Wang, Q. R.; Cui, Y. S. ; Liu, X. M. ; Dong, Y. T. ; Christie, P. ; J. Environ. Sci. H., Part A: - Toxic/H. Subst. \& Environ. Eng. 2003, 38, 174.

14. Sperling, M. von; Costa, A. M. L. M. da; Castro, A.; Manual de saneamento e proteção ambiental para os municípios, DESA/UFMG: Belo Horizonte, 1995. 
15. Amaral Sobrinho, N. M. B.; Costa, L. M.; Oliveira, C. de; Velloso, A. C. X.; R. bras. Ci. Solo 1992, 16, 271.

16. Moura, M. C. S. de; Lopes, A. N. C.; Moita, G. C.; Moita Neto, J. M.; Quim. Nova 2006, 29, 429.

17. http://www.epa.gov/SW-846/pdfs/3050b.pdf, acessada em Março 2005.

18. Campos, M. L.; Silva, F. N. da; Neto, A. E. F.; Guilherme, L. R. G.; Marques, J. J.; Antunes, A. S.; Pesq. agropec. bras. 2005, 40, 361.

19. Anda - Associação Nacional para Difusão de Adubos e Corretivos Agrícolas; Análise de corretivos agrícolas, Nagy: São Paulo, 1989.

20. Oliveira, E. L.; Parra, M. S.; R. bras. Ci. Solo 2003, 27, 171.

21. Albuquerque, J. A.; Argenton, J.; Fontana, E. C.; Costa, F. S.; Rech, T. D.; $R$. bras. Ci. Solo 2002, 26, 1065.

22. Nelson, P. N.; Baldock, J. A. ; Oades, J. ; Aust. J. Soil R. 1998, 36, 883.

23. Albuquerque, J. A.; Bayer, C.; Ernani, P. R. ; Fontana, E. C. ; R. bras. Ci. Solo 2000, 24, 295

24. Meurer, E. J.; Fundamentos de química do solo, $3^{\mathrm{a}}$ ed., Evangraf: Porto Alegre, 2006.

25. van Olphen, H.; An introduction to clay colloid chemistry, $2^{\text {nd }}$ ed., John Wiley \& Sons: New York, 1977

26. Oliveira, F. C.; Marques, M. O.; Bellingieri, P. A.; Perecin, D.; Sci. Agric. 1995, 52,123 .
27. Ernani, P. R.; Bittencourt, T. P.; Bidaje, V. ; Cristani, J. ; R. bras. Ci. Solo 2001 , 25, 905.

28. Galdos, M. V.; Maria, I. C. de; Camargo, O. A.; R. bras. Ci. Solo 2004, $28,569$.

29. Silva, C. A.; Rangel, O. J. P.; Dynia, J. F.; Bettiol, W.; Manzatto, C. V.; R. bras. Ci. Solo 2006, 30, 353 .

30. Cetesb - Companhia de Tecnologia de Saneamento Ambiental; Relatório de estabelecimento de valores orientadores para solos e águas subterrâneas no Estado de São Paulo, São Paulo, 2001.

31. Cetesb - Companhia de Tecnologia de Saneamento Ambiental; Aplicação de biossólidos de sistemas de tratamento biológico em áreas agrícolas-Critérios para projeto e operação: manual técnico, São Paulo, 1999.

32. Pombo, L. C. A.; R. bras. Ci. Solo. 1996, 20, 423.

33. Canaverolo, S. V. Jr.; Técnicas de Caracterização de Polímeros, Artliber: São Paulo, 2003.

34. Bellamy, L. J.; The Infrared Spectra of Complex Molecules, $3^{\text {rd }}$ ed., Chapman and Hall: New York, 1975

35. Ernani, P. R.; Barber, S. A.; R. bras. Ci. Solo 1993, 17, 41.

36. Skoog, D. A.; Holler, F. J.; Nieman, T. A.; Princípios de análise instrumental, $5^{\text {a }}$ ed., Bookman: Porto Alegre, 2002. 